\title{
Avaliação in vitro da resistência de duas resinas compostas submetidas a teste de compressão
}

In vitro evaluation of the strength of two composite resins subjected to compression test Evaluación in vitro de la resistencia de dos resinas compuestas sometidas a prueba de compresión

\author{
Mirla Lays Dantas de ALMEIDA ${ }^{1}$ \\ Gymenna Maria Tenório GUÊNES ${ }^{2}$ \\ Luanna Abílio Diniz Melquiades de MEDEIROS ${ }^{3}$ \\ Mylena de Araújo RÉGIS ${ }^{1}$ \\ Marcus Vinícius Lia FOOK ${ }^{4}$ \\ Darlene Cristina Ramos Eloy DANTAS ${ }^{5}$ \\ Danúbia Roberta Medeiros NÓBREGA
}

\begin{abstract}
IGraduada em Odontologia pela Universidade Federal de Campina Grande (UFCG), Unidade Acadêmica de Ciências Biológicas (UACB) 48708-110, Patos-PB, Brasil
${ }^{2}$ Professora Doutora, Disciplinas Pré clínica Multidisciplinar II, Pré clínica Multidisciplinar III e Clínica multidisciplinar I Curso de Odontologia da Universidade Federal de Campina Grande (UFCG), Unidade Acadêmica de Ciências Biológicas (UACB) 58708-110 Patos-PB, Brasil

${ }^{3}$ Professora Doutora, Disciplinas de Clínica Multidisciplinar I e III, Estágio em Clínica Multidisciplinar I e Pré-Clínica Multidisciplinar V do curso de Odontologia da Universidade Federal de Campina Grande (UFCG), Unidade Acadêmica de Ciências Biológicas (UACB)58708-110, Patos-PB, Brasil

${ }^{4}$ Professor Doutor do Programa de Pós-Graduação em Ciência e Engenharia de Materiais/DEMa/UFCG, Bolsista de Produtividade do CNPq, Coordenador do Laboratório CERTBIO/DEMa/Universidade Federal de Campina Grande (UFCG), 58429-900, Campina Grande-PB, Brasil

${ }^{5}$ Coordenadora do Projeto Banco de Dentes Humanos da UEPB Campus I (BDH/UEPB), Professora da Disciplina de Dentística da Universidade Estadual da Paraíba (UEPB), 58429-600, Campina Grande-PB, Brasil

${ }^{6}$ Curso de Odontologia das Faculdades Integradas de Patos (FIP), 58704-000 Patos-PB, Brasil
\end{abstract}

\section{Resumo}

Introdução: $\mathrm{O}$ aumento da demanda pela odontologia estética guiou o desenvolvimento de resinas compostas com melhores propriedades físicas e mecânicas. Por isso, percebe-se que há atualmente disponibilidade de uma grande variedade desse material. Dessa forma faz-se necessário a realização de estudos científicos acerca das resinas composta. Objetivo: Avaliar comparativamente, in vitro, à resistência de duas resinas compostas fotopolimerizáveis encontrados no mercado: a Filtek Z350 XT da 3M e a Zirconfill da Technw, após teste mecânico de compressão e análise estatística dos resultados. Material e método: Quatro amostras de cada resina composta foram submetidas ao teste de resistência à compressão numa máquina de ensaio universal. Os resultados foram analisados estatisticamente através do teste de MannWhitney com margem de erro 5.0\%. Para digitar os dados e obter os cálculos estatísticos foi utilizado o programa IBM SPSS na versão 23. Resultados: A média da força foi mais elevada na resina Zirconfill do que na resina Z350; entretanto, a mediana foi mais elevada na resina Z350. Para a margem de erro fixada $(5,0 \%)$ não há comprovação de diferença significativa $(p>0,05)$ entre as duas resinas em relação à força de resistência analisada. Conclusão: As resinas compostas testadas mostram valores de resistência a compressão semelhantes e não apresentam diferenças relevantes.

Descritores: Resinas Compostas; Nanotecnologia; Odontologia.

\section{Abstract}

Introduction: The increased demand for aesthetic dentistry guided the development of composite resins with better physical and mechanical properties. Therefore, it is perceived that there is currently a wide variety of this material available. In this way it is necessary to carry out scientific studies on composite resins. Objective: To evaluate in vitro the resistance of two photopolymerizable composite resins found in the market: Filtek Z350 XT from 3M and Zirconfill from Technw, after mechanical compression test and statistical analysis of the results. Material and method: Four samples of each composite resin were submitted to the compressive strength test in a universal test machine. The results were statistically analyzed using the Mann-Whitney test with a margin of error of $5.0 \%$. To enter the data and obtain the statistical calculations, the IBM SPSS program in version 23 was used. Results: Mean strength was higher in Zirconfill resin than in Z350 resin; however, the median was highest in the Z350 resin. For the fixed margin of error (5.0\%) there was no significant difference (p>0.05) between the two resins in relation to the strength of resistance analyzed. Conclusion: The composite resins tested showed similar values of compressive strength and presented no relevant differences.

Descriptors: Composite Resins; Nanotechnology; Dentistry.

\section{Resumen}

Introducción: El aumento de la demanda por la odontología estética guió el desarrollo de resinas compuestas con mejores propiedades físicas y mecánicas. Por eso, se percibe que hay actualmente disponibilidad de una gran variedad de ese material. De esta forma se hace necesaria la realización de estudios científicos sobre las resinas compuesta. Meta: Evaluar comparativamente, in vitro, a la resistencia de dos resinas compuestas fotopolimerizables encontradas en el mercado: la Filtek Z350 XT de 3M y la Zirconfill de Technw, después de la prueba mecánica de compresión y análisis estadístico de los resultados. Material y método: Cuatro muestras de cada resina compuesta se sometieron al ensayo de resistencia a la compresión en una máquina de ensayo universal. Los resultados fueron analizados estadísticamente a través de la prueba de Mann-Whitney con margen de error 5.0\%. Para escribir los datos y obtener los cálculos estadísticos se utilizó el programa IBM SPSS en la versión 23. Resultados: La media de la fuerza fue más elevada en la resina Zirconfill que en la resina Z350; sin embargo, la mediana fue más elevada en la resina Z350. Para el margen de error fijado $(5,0 \%)$ no hay comprobación de diferencia significativa ( $p>0,05)$ entre las dos resinas en relación a la fuerza de resistencia analizada. Conclusión: Las resinas compuestas probadas muestran valores de resistencia a compresión similares y no presentan diferencias relevantes.

Descriptores: Resinas Compuestas; Nanotecnología; Odontología.

\section{INTRODUÇÃO}

Vários avanços em pesquisas científicas e tecnologia proporcionaram incontáveis benefícios a odontologia. Desde que Bowen ${ }^{1}$ apresentou a sociedade odontológica um compósito à base de BISGMA (Bisfenol A glicidil metacrilato) e partículas de cargas silanizadas, as resinas passaram por algumas modificações para melhoria de suas propriedades físicas e de seu desempenho clínico, principalmente no que diz respeito ao tamanho de suas partículas, que, atualmente, graças à nanotecnologia, são partículas nanométricas; apesar de ainda apresentarem a mesma formulação elaborada por Bowen $^{1,2}$.

Com o aumento da procura por


procedimentos estéticos por parte dos pacientes, verificou-se a necessidade de se realizar restaurações que se aproximam cada vez mais dos aspectos dos dentes naturais e que utilizem materiais que atendam aos requisitos físicos e mecânicos exigidos, tais como: biocompatibilidade, resistência as forças de mastigação, adaptação marginal, entre outros. Para a realização desses procedimentos, o profissional de odontologia tem ao seu dispor uma grande quantidade de materiais que possibilitam a execução de várias técnicas restauradoras ${ }^{3}$.

Por isso, graças às suas excelentes propriedades estéticas e também as propriedades mecânicas e biológicas, as resinas compostas passaram a ser utilizadas também, nas restaurações de dentes posteriores, já que proporcionam um procedimento mais conservador, evitando desgaste dental e restringindo, tanto o preparo cavitário como a remoção do tecido cariado ${ }^{4}$.

Junto ao fato das inúmeras pesquisas terem melhorado significativamente as resinas compostas e devido as restaurações com esse tipo de material representarem uma das atividades mais desenvolvidas no consultório odontológico, a competição entre os fabricantes é cada vez maior, o que explica o fato de existir uma vasta quantidade de resinas compostas disponíveis no mercado.

Portanto, em virtude do caminho estético que a odontologia vem trilhando e do crescente número de resinas compostas atualmente presentes no mercado, a proposta desse trabalho é avaliar comparativamente, in vitro, à resistência a compressão e a tração de dois materiais restauradores estéticos encontrados no mercado; as resinas compostas fotopolimerizáveis: Filtek Z350 XT da $3 \mathrm{M}$ e a Zirconfill da Technw.

\section{MATERIAL E MÉTODO}

O estudo foi realizado por meio da utilização de oito terceiros molares humanos hígidos, que foram extraídos por indicação cirúrgica; tais dentes foram obtidos a partir de doação do Projeto de Extensão Banco de Dentes Humanos da Universidade Estadual da Paraíba- Campus I (Campina Grande-PB).

Após a seleção prévia dos elementos utilizados, eles foram limpos com uma cureta McCall 13-14 (Golgran-Millenium, São Paulo, Brasil) e uma escova de cerdas Robinson (Microdont, São Paulo, Brasil) com pasta de pedra-pomes e água para remover os possíveis tecidos moles e detritos presentes. Os dentes foram desinfectados em solução de clorexidina 2\% (Maquira, Maringá, Brasil) por 30 minutos e preparados para armazenagem em solução salina a $0,9 \%$ sob refrigeração, que era substituída por uma solução fresca uma vez por semana até o início da parcela experimental do estudo (Figuras 1 a 3). A armazenagem não ultrapassou um período de 6 meses.
Os dentes foram então divididos igualmente em dois grupos $(n=4)$. No grupo 1 foram feitas as restaurações usando a resina Filtek Z350 XT (3M ESPE, St Paul, MN, EUA) e no grupo 2 as restaurações foram realizadas com a resina composta Zirconfill (Technw). Todos os elementos dentais dos dois grupos foram submetidos ao teste de compressão.

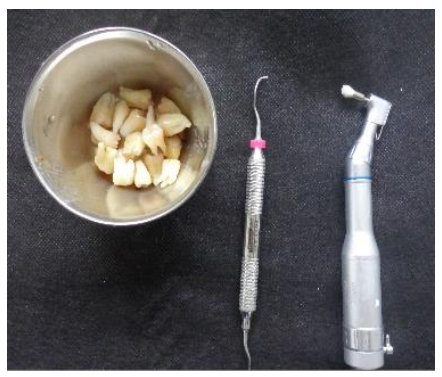

Figura 1: Limpeza e desinfecção dos elementos dentários: cureta McCall 13-14 e escova de cerdas Robinson utilizadas na limpeza dos dentes.

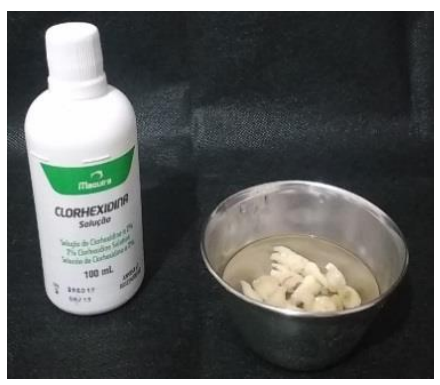

Figura 2: Desinfecção dos elementos dentários com solução de clorexidina $2 \%$.

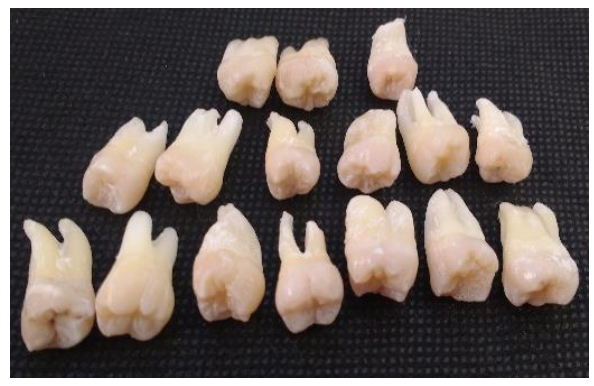

Figura 3. Elementos dentários prontos para a armazenagem.

Padronizou-se cavidades do tipo classe V que foram confeccionadas por apenas um operador, na Clínica Escola de Odontologia da Universidade Federal de Campina Grande, campus Patos - PB. As cavidades foram realizadas na superfície vestibular dos terceiros molares, por meio de broca cilíndrica diamantada $\mathrm{n}^{\circ} 3100$ (KG Sorensen, São Paulo - SP, Brasil) sob refrigeração constante e padronizadas (Figuras 4 a 6) em 3,0 mm para a altura oclusogengival, 3,0 mm para extensão mésio-distal e 1,5 $\mathrm{mm}$ de profundidade axial. A margem oclusal ficou estabelecida em esmalte e a margem gengival em dentina ${ }^{5}$.

Ambos os grupos sofreram tratamento com o sistema adesivo Single Bond Universal (3M ESPE / St Paul, MN, EUA), precedidos de corrosão ácida de dentina com $37 \%$ de ácido fosfórico ÁCIODO - P GEL (Allplan, São Paulo - SP, Brasil) por 15 segundos e no esmalte por 30 segundos, seguido de enxaguamento com pulverização de ar / água de uma seringa tripla por 15 segundos e secos com bolinhas 
de algodão, para obter uma superfície de dentina úmida e brilhante. $\mathrm{O}$ adesivo foi então aplicado em duas camadas por meio de um aplicador microbrush e o excesso desse material, após a primeira aplicação, foi removido também com o auxílio de microbrush (limpo), então aconteceu a fotopolimerização utilizando uma unidade LED de cura de luz, LEDX T 2400 (ORTHOMETRIC, Marília - SP, Brasil) com intensidade de luz $\left(720 \mathrm{~mW} / \mathrm{cm}^{2}\right)$ que foi verificada com o auxílio do radiômetro presente no próprio equipamento LEDEX - T 2400 antes do início dessa etapa laboratorial, por um período de 20 segundos. Após a aplicação do sistema adesivo, seguiu-se a restauração das cavidades dos elementos dentários.

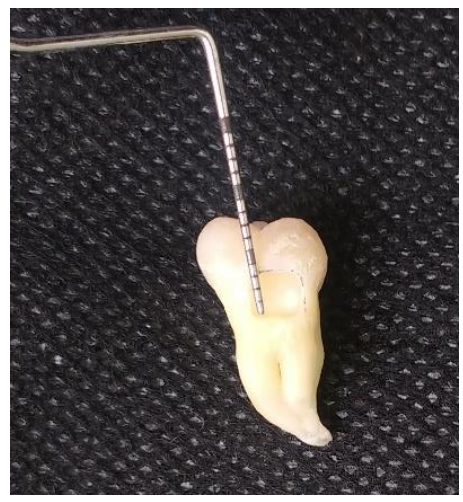

Figura 4: Padronização das cavidades: altura ocluso-gengival.

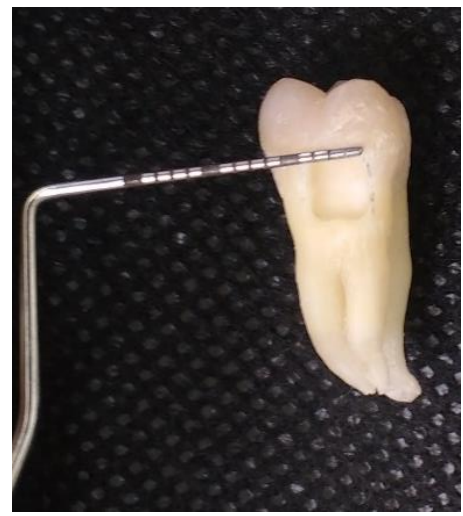

Figura 5: Padronização das cavidades: extensão mésio-distal.

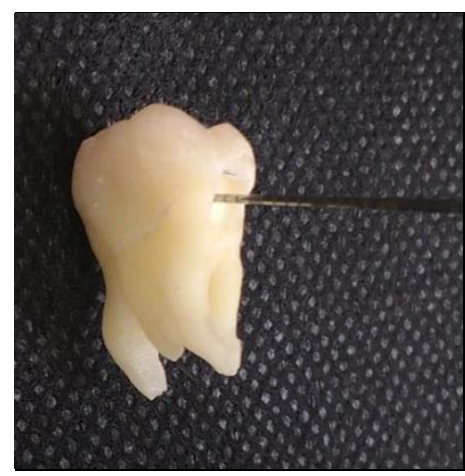

Figura 6: Padronização das cavidades: profundidade axial.

As resinas foram aplicadas em incrementos de aproximadamente $2 \mathrm{~mm}$ até completo preenchimento da cavidade. Cada incremento de resina foi polimerizado durante 20 segundos e por mais 20 segundos no final da restauração; utilizandose a unidade LED de cura de luz, LEDEX - T 2400 (ORTHOMETRIC, Marília - SP, Brasil) as intensidades de luz foram aferidas por meio do radiômetro no início $\left(810 \mathrm{~mW} / \mathrm{cm}^{2}\right)$ e no fim $(730$ $\mathrm{mW} / \mathrm{cm}^{2}$ ) das restaurações.

$\mathrm{O}$ excesso de material restaurador foi removido 24 horas após a realização da restauração, com ponta de acabamento FG 3168F (KG Sorensen, São Paulo-SP, Brasil) e o polimento das restaurações, feito com discos abrasivos Sof-Lex (3M ESPE, São Paulo - SP, Brasil) com diâmetro de 19,05 mm em ordem decrescente de granulação ${ }^{5}$.

Os dentes assim preparados foram armazenados em solução salina a $0,9 \%$ sob refrigeração até o momento do ensaio mecânico.

Seguiu-se a etapa laboratorial, que foi realizada no CERTBIO - Laboratório de Avaliação e Desenvolvimento de Biomateriais do Nordeste UFCG - onde a compressão axial foi realizada em uma máquina de ensaio universal (modelo 3366, Instron) que contém dois pratos de compressão. Dessa foram a face restaurada do dente ficou constantemente em contato com um dos pratos (Figura 7) que aplicaram uma força de até $10.000 \mathrm{~N} \mathrm{a}$ uma velocidade de cruzamento de $0,5 \mathrm{~mm} / \mathrm{min}$. A força de compressão foi aplicada até que ocorresse a fratura do elemento dentário e o valor de tal força é então registrado em Newtons e convertido em Megapascal.

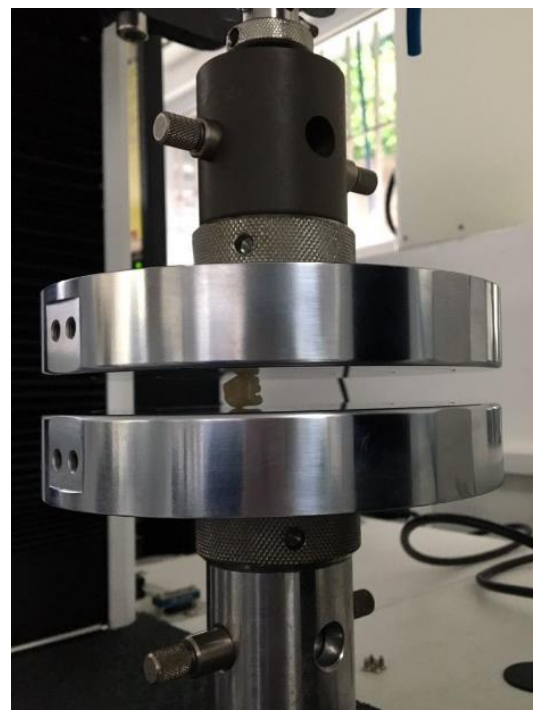

Figura 7: Padronização.

Os dados de resistência à fratura foram então submetidos à análise estatística para comparação entre os grupos por meio do teste de Mann-Whitney. $\mathrm{O}$ referido teste foi escolhido devido ao número de amostras utilizadas.

A margem de erro utilizada na decisão do teste estatístico foi $5.0 \%$ e o programa IBM SPSS na versão 23 foi utilizado para digitar os dados e obter os cálculos estatísticos.

\section{RESULTADOS}

A Tabela 1 exibe os resultados da força de resistência à compressão entre as duas resinas analisadas: Zirconfill e Z350. Estes foram expressos 
através das estatísticas: média, desvio padrão, coeficiente de variação e mediana.

Destaca-se que: a média (Gráfico 1) da força foi mais elevada na resina Zirconfill (7,36 Mpa) do que na resina Z350 (5,58 Mpa); entretanto a mediana foi mais elevada na resina Z350 (6,10 Mpa) contra 5,88 Mpa da Zirconfill. Para a margem de erro fixada $(5,0 \%)$ não se comprova diferença significativa $(p>$ 0,05 ) entre as duas resinas em relação à força de resistência analisada.

Com relação à variabilidade, expressa através do coeficiente de variação (Tabela 1), esta se mostrou elevada na resina Zirconfill que teve valor igual a $91,58 \%$ (superior a 50,0\%) e foi reduzida na resina Z350 (coeficiente de variação igual a 20,14 $<33,3 \%)$.

Tabela 1. Análise estatística da força de resistência à compressão segundo a resina utilizada

\begin{tabular}{|c|c|c|c|}
\hline Análise Estatística & \multicolumn{2}{|c|}{ Resina } & Valor de $\mathbf{p}^{*}$ \\
\hline & Zirconfil & Z350 & \multirow{5}{*}{0,886} \\
\hline Média** & 7,36 & 5,58 & \\
\hline Desvio padrão** & 6,74 & 1,57 & \\
\hline Coeficiente de variação (\%) & 91,58 & 20,14 & \\
\hline Mediana $^{* * *}$ & 5,88 & 6,10 & \\
\hline
\end{tabular}

*Teste de Mann-Whitney

**Medidas em Mpa

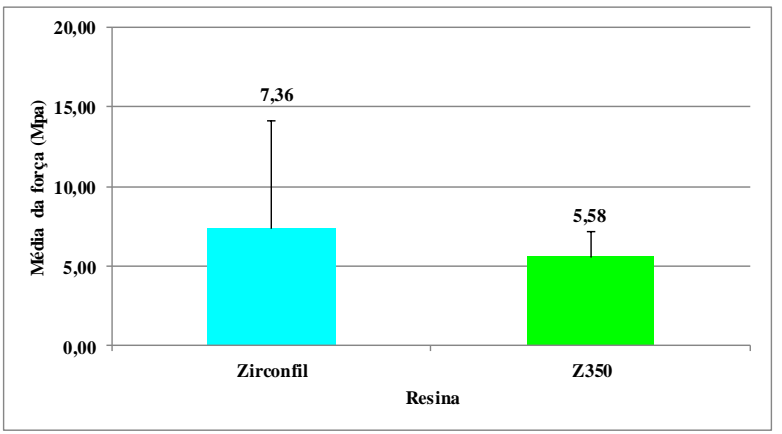

Gráfico 1: Média da força de resistência à compressão segundo a resina utilizada.

\section{DISCUSSÃO}

$\mathrm{Na}$ busca por avanços, desde a sua introdução no mercado, os compósitos resinosos sofreram várias transformações em sua composição. Alterações aconteceram nas partículas de carga inorgânica; onde houve redução nos seus tamanhos; mudança no formato, na distribuição e na morfologia destas. Outras alterações foram feitas na matriz orgânica das resinas compostas ${ }^{6,7}$.

Com isso, nota-se que as pesquisas científicas consolidaram que as possíveis melhorias das resinas compostas estão relacionadas a alterações em seu componente de carga inorgânica, principalmente no que diz respeito ao material utilizado como carga ${ }^{6,7}$.

Por sua vez, a nanotecnologia objetivou desenvolver resinas compostas que poderiam ser usadas tanto em dentes anteriores como nos posteriores. Que tivessem um alto polimento inicial e grande capacidade de retenção deste, características essas já apresentadas pelas resinas compostas microparticuladas. Também era objetivo destas resinas que elas apresentassem propriedades mecânicas excelentes capazes de suportar altas cargas de estresse interoclusais; típico perfil das resinas compostas micro-híbridas. Atualmente percebe-se que as resinas compostas nanoparticuladas são capazes de fornecer excelente resistência ao desgaste, longevidade e estética, devido a sua excelente capacidade de polimento, conservação deste e aparência lustrosa ${ }^{8}$.

A resina composta Zirconfill, nano-hibrida, mostra em sua composição cargas de sílicas convencionais já consagradas, em uma associação com a zircônia e a diatomita, que é uma sílica porosa.

Segundo o seu fabricante; em todas as análises, a resina Zirconfill apresentou-se mais resistente à compressão, do que todos os outros materiais de mesma aplicação clínica, atualmente disponíveis no mercado e já testados conforme os dados trazidos pela literatura dos próprios fabricantes e, segundo esses, por testes independentes de pesquisadores.

No entanto esse padrão não foi observado no presente estudo. Visto que foi notado não haver diferença significativa entre as duas resinas compostas analisadas.

Mota et al. ${ }^{9}$ pontuam que ao se realizar estudos com compósitos de mesma classificação, almeja-se que eles manifestem comportamentos mecânicos similares, corroborando com que ocorre no presente exposto; visto que não houve diferenças significativas entre as resinas compostas testadas.

Também concorda com Rosa ${ }^{10}$ quando conclui que as resinas compostas testadas atingiram médias estatísticas similares para resistência à compressão.

Os valores de resistência à compressão, obtidos no presente trabalho variados entre 7,36 Mpa para a resina Zirconfill (nano-híbrida) e 5,58 Mpa para a Z350 (nanoparticulada) não corroboraram com os valores de Souza e colaboradores ${ }^{11}$ que realizaram teste de resistência à compressão na resina composta nanoparticulada Filtek Supreme XT e pontuou o valor médio desta em 248,2 Mpa. Mota et al. ${ }^{9}$ encontraram valor de 184,67 Mpa para essa mesma resina composta, afastando-se dos resultados desse trabalho para a resina nanoparticulada.

Provavelmente este fato é explicado devido às diferenças no tamanho das amostras, na velocidade do cruzamento, força aplicada e no meio de armazenamento das mesmas. Ou seja, múltiplas variáveis podem interferir na resistência mecânica das resinas compostas ${ }^{9}$.

Contudo, a hipótese de que as resinas compostas testadas neste trabalho (Z350 e Zirconfill) teriam comportamentos mecânicos similares no teste proposto, foi admitida. E sugere-se que outros estudos laboratoriais sejam realizados para que outras propriedades sejam avaliadas; tais como: 
CONFLITO DE INTERESSES

Os autores declaram não haver conflitos de interesse. superficial.

\section{CONCLUSÃO}

Com base na metodologia utilizada e nos resultados obtidos nessa pesquisa, é possível concluir que em relação a resistência a compressão, estatisticamente, ambas as resinas compostas testadas mostram valores de resistência semelhantes e que, portanto, não apresentam diferenças relevantes.

\section{REFERÊNCIAS}

1. Bowen RL. Use of epoxy resins in restorative materials. J Dent Res. 1956;35(3):360-69.

2. Chagas LS. Avaliação da resistência flexural e módulo de elasticidade de uma resina do tipo Bulk Fill em comparação com uma resina convencional [monografia de conclusão de curso]. Santa Cruz do Sul: Universidade de Santa Cruz do Sul UNISC; 2016.

3. Behr M, Proff P, Kollbeck C, Langriegr S, Kunze $\mathrm{J}$, Handel $\mathrm{G}$ et al. The bond strength of the resinto-zirconia interface using diferente bonding concepts. J Mech Behav Biomed Mater. 2011;4(1):2-8.

4. Rodrigues DS. Análise da porosidade, resistência mecânica e desgaste de cimentos de ionômero de vidro e resina composta [dissertação]. Porto: Faculdade de Ciências da Saúde, Universidade Fernando Pessoa; 2014.

5. Pereira AFV, Miranda Junior WG. Avaliação in vitro da microinfiltração e adaptação marginal em restaurações classe $\mathrm{v}$ simulando lesões de abfração: estudo piloto. Arq Cent Estud Curso Odontol Univ Fed Minas Gerais. 2008;44(2):18-25.

6. Ferracane JL. Resin composite - state of the art. Dent Mater. 2011; 27(1):29-38.

7. Han JM, Lin H, Zheng G, Shinya A, Gomi H, Shinya A et al. Effect of nanofiller on wear resistance and surface roughness of resin composites. Chin J Dent Res. 2012;15(1):41-7.

8. Mitra SB, Wu D, Holmes B. An application of nanotechnology in advanced dental materials. J Am Dent Assoc. 2003;134(10):1382-90.

9. Mota EG, Weiss A, Spohr AM, Oshima HMS, Carvalho LMN. Relationship between filler content and selected mechanical properties of six microhybrid composites. Rev odonto cienc. 2011;26(2):151-55.

10.Rosa RS. Propriedades mecânicas de resinas compostas com nanopartículas [tese]. Porto Alegre: Pontifícia Universidade Católica do Rio Grande do Sul, Faculdade de Odontologia; 2010.

11. Souza ALT, Oliveira FMMPC, Moysés MR, Assis CPD, Ribeiro JCR, Dias SC. Avaliação da resistência à compressão de resinas compostas fotopolimerizáveis. Arq Odontol. 2007;43(1):30-5.
Submetido em 19/09/2018
Aceito em 11/11/2018

Submetido em 19/09/2018
Aceito em 11/11/2018

\section{AUTOR PARA CORRESPONDENCIA}

\section{Mirla Lays Dantas de Almeida}

mirlalays2@hotmail.com 